\title{
Insulin-Like Growth Factor-1 and Osteocalcin Are Correlated with Markers of Osteoporosis in Postmenopausal Women with Type-2 Diabetes
}

\author{
Maher A. Kamel ${ }^{1}$, Madiha H. Helmy ${ }^{1}$, Anna N. Abou Rayah ${ }^{2}$, Nevine Mohannad ${ }^{2}$, Huda M. N. Hania ${ }^{1}$ \\ ${ }^{1}$ Department of Biochemistry, Medical Research Institute, Alexandria University, Alexandria, Egypt \\ ${ }^{2}$ Department of Internal Medicine, Faculty of Medicine, Alexandria University, Alexandria, Egypt \\ Email: maher.kamel@alexu.edu.eg
}

Received June 17, 2013; revised July 15, 2013; accepted August 13, 2013

Copyright (C) 2013 Maher A Kamel et al. This is an open access article distributed under the Creative Commons Attribution License, which permits unrestricted use, distribution, and reproduction in any medium, provided the original work is properly cited.

\begin{abstract}
Objective: The study was conducted to evaluate the relation between insulin-like growth factor-1 and osteocalcin and markers of bone modulation (osteoprotegerin; OPG, receptor activator nuclear kappa B; RANK and RANK ligand; RANKL) in postmenopausal Type 2 diabetic women with and without osteoporosis. Methods: The study was conducted on 90 female divided into three groups (30 each). Group I included healthy postmenopausal women as a control, Group II included diabetic postmenopausal women without osteoporosis Group III included diabetic postmenopausal women with osteoporosis. Fasting blood samples were obtained for the determination of blood glucose, HbA1c, total and ionized calcium, OPG, RANK and RANKL. Also the levels of IGF-1 and osteocalcin were assessed. Results: In postmenopausal Type 2 diabetic women, the osteoporosis resulted in derangement in OPG/sRANKL system. The serum level of OPG was elevated while sRANKL declines in osteoporotic postmenopausal Type 2 diabetic women. IGF-1 level decreased in diabetic postmenopausal women but those women with osteoporosis showed a great decline by about $60 \%$. IGF-1 level in osteoporotic diabetic postmenopausal women was correlated with BMD and most bone turnover markers (OPG, sRANKL, OPG/sRANKL). Osteocalcin declined significantly only in those women with osteoporosis not without osteoporosis. Conclusions: The circulating levels of OPG and sRANKL were not useful markers for bone status in postmenopausal women while the circulating levels of IGF-1 and osteocalcin might give useful information about bone status in postmenopausal diabetic women.
\end{abstract}

Keywords: Osteoporosis; Type 2 Diabetes; Postmenopause; IGF-1; Osetocalcin; OPG; RANK

\section{Introduction}

Osteoporosis, a global age-related health problem in both male and female elderly, insidiously deteriorates the microstructure of bone, particulaly at trabecular sites, such as vertebrate, ribs and hips, culminating in fragility fractures, pain and disability [1].

The process of bone modeling and remodeling is vital for bone health. The bone remodeling unit is composed of osteoblasts, which form bone, and osteoclasts, which break down bone [2]. The osteoblast and osteoclast differentiation and activation depend on a family of biologically related TNF/TNF-like proteins: osteoprotegerin (OPG), receptor activator of nuclear factor (NF) $\kappa \mathrm{B}$ (RANK), and RANK ligand (RANKL). RANKL accelerates osteoclastogenesis when it binds to its receptor RANK on osteoclast precursor cells to enhance nuclear factor-kappa B (NF- $\kappa \mathrm{B})$ and other signaling pathways which promote osteoclast formation, activation, and survival [3].

OPG, which is produced by osteoblasts, is a natural decoy receptor for RANKL by sequestering RANKL and neutralizing its effects. The OPG/RANKL/RANK system plays a significant role in postmenopausal osteoporosis. Postmenopausal women express higher levels of RAN$\mathrm{KL}$ on marrow stromal cells or lymphocytes than premenopausal women or postmenopausal women taking estrogen $[3,4]$.

Insulin-like growth factor-1 (IGF-1) produced mainly by liver and locally by osteoblast, is one of the most abundant growth factors presents in bone that stimulates osteoblast activity, subsequently leading to bone matrix formation and inhibition of bone collagen degradation $[5,6]$. Also, osteocalcin, the second most abundant pro- 
tein in bone after collagen, is secreted by osteoblast and thought to participate in mineralization and calcium ion homeostasis [7]. Also experimental studies revealed new metabolic function as hormone, being involved in blood glucose regulation, insulin secretion and in adipose tissue structure [8].

Osteoporosis and Type 2 diabetes mellitus (DM) are usually viewed as separate disease that associated with aging. However accumulating evidences indicate that these diseases are interrelated $[9,10]$. Some studies were suggested a possible increased risk of osteoporosis in patients with either Type 1 or Type $2 \mathrm{DM}$, but this association remains controversial and conflicting, especially in Type 2 [11-13]. Although bone mineral density (BMD) is considered as a gold standard for evaluating fracture risk in non-DM osteoporosis, it is not useful for assessing fracture risks in Type 2 DM [9]. Therefore, a diagnostic marker other than BMD needs to be explored. IGF-1 and osteocalcin seem to be two of candidate's markers that may replace the insensitivity of BMD in assessing the osteoporosis in Type 2 DM patients. So this study was conducted to evaluate osteoporosis in postmenopausal women with Type 2 DM and to explore IGF-1 and osteocalcin as a markers for assessing bone remodeling in those subjects.

\section{Subjects and Methods}

\subsection{Subjects}

After the acceptance of the ethical community of the Medical Research Institute-Alexandria University, the study was conducted on 90 female divided into three groups (30 each). The first include apparently healthy postmenopausal women as a control (Group I), the second include diabetic postmenopausal women without osteoporosis (Group II) and the third include diabetic postmenopausal women with osteoporosis (Group III). An informed consent was obtained from all the participants in the study.

Exclusion criteria were malignant diseases, hyperthyroidism, hyperparathyroidism, osteomalacia, renal and hepatic diseases, chronic treatment with antiacids, estrogen, adrenal or anabolic steroids, anticonvulsants, anticoagulants, or pharmacologic doses of vitamin $\mathrm{A}$ and $\mathrm{D}$ supplements 6 months prior to the study. Also, history of unstable cardiovascular diseases or uncontrolled hypertension was excluded.

Venous blood samples were collected after overnight fasting, serum samples were rapidly separated by centrifugation at $3500 \mathrm{rpm}$ for $10 \mathrm{~min}$ and stored at $-20^{\circ} \mathrm{C}$.

\subsection{Methods}

\subsubsection{BMD Measurements}

Dual X-ray absorptiometric (DXA) measurements of areal BMD $\left(\mathrm{g} / \mathrm{cm}^{2}\right)$ at the lumbar spine L1-L4) and the femoral neck were carried out using a Lunar DPX Prodigy densitometer (Lunar, Madison, WI, USA). Daily standardized measurement of a Lunar phantom and Hologic Anatomic spine phantom served as a quality control for the bone densitometer.

\subsubsection{Biochemical Assays}

Fasting glucose was determined by the glucose oxidase method [14] and Glycated haemoglobin (HbAlc) was determined using a turbidimetric inhibition immunoassay for haemolysed whole blood [15]. Serum phosphorus, total and ionized calcium were assessed [16]. IGF-I was assayed using ELISA kit (R \& D Systems, Inc, USA), Osteocalcin ELISA kit was purchased from (Diagnositc Systems Laboratories, USA). Osteoprotegerin and soluble RANKL were detected by ELISA kits (RayBiotech Inc, USA).

\subsubsection{Statistical Analysis}

The results obtained from this work, was statistically analyzed by statistical package calculated parameters for the Social Sciences (SPSS) program version 15.0. All data are presented as Mean \pm SEM. A one way analysis of variance (ANOVA) was performed on each variable. The correlations between variables were carried out by Pearson coefficient. The critical value for significance was $p<0.05$.

\section{Results}

The demographic and clinical data of the studied groups are summarized in Table 1. The diabetic osteoporotic postmenopausal women (Group III) appear to be of higher age, weight and BMI compared to those non-osteoporotic diabetic postmenopausal (Group II) and control women (Group I). The fasting blood glucose levels in diabetic women is significantly elevated by about $91 \%$ in group II and about $161 \%$ in group III compared to control value. Also elevated levels of glycosylated hemoglobin were significantly elevated in diabetic groups especially in group III compared to control which indicated that the control of blood levels in those patients over the last two months is not correctly controlled. The result showed that the total calcium levels were slightly decreased in diabetic group by about 6 and 10\% in group II and III; respectively compared to control subjects. The ionized calcium and phosphorus showed no significant change in both diabetic groups (Table 1).

The osteoprotegerin level in diabetic postmenopausal women with osteoporosis is highly elevated by about $96 \%$ and by about $20 \%$ in those without osteoporosis compared to control group (Figure 1). However, no significant changes were observed in sRANKL levels, while group III showed a slight non-significant decrease by 
Table 1. Demographic and clinical data of the control subjects and diabetic postmenopausal women with and without osteoporosis.

\begin{tabular}{|c|c|c|c|}
\hline & \multirow{2}{*}{$\begin{array}{c}\text { Control Group I } \\
\quad(\mathbf{n}=\mathbf{3 0})\end{array}$} & \multicolumn{2}{|c|}{ Diabetic groups } \\
\hline & & Without osteoporosis Group II $(\mathbf{n}=\mathbf{3 0})$ & With osteoporosis Group III $(n=30)$ \\
\hline Age (years) & $49.13 \pm 5.96$ & $50.73 \pm 4.86$ & $62.66 \pm 5.39^{*, \#}$ \\
\hline Height (cm) & $165.30 \pm 4.43$ & $164.03 \pm 3.85$ & $162.33 \pm 3.17$ \\
\hline Weight (kg) & $66.16 \pm 3.57$ & $68.43 \pm 3.05^{*}$ & $71.36 \pm 3.46^{*, \#}$ \\
\hline BMI $\left(\mathrm{Kg} / \mathrm{m}^{2}\right)$ & $24.64 \pm 1.61$ & $25.07 \pm 1.29$ & $26.65 \pm 1.64$ \\
\hline FBG (mg/dl) & $86.37 \pm 8.56$ & $165.29 \pm 24.19^{*}$ & $225.6 \pm 25.14^{* \#}$ \\
\hline Hemoglobin A1c & $5.78 \pm 1.13$ & $8.47 \pm 0.5^{*}$ & $9.64 \pm 0.71^{*, \#}$ \\
\hline $\mathrm{Ca}(\mathrm{mg} / \mathrm{dl})$ & $9.32 \pm 0.71$ & $8.72 \pm 0.46^{*}$ & $8.34 \pm 0.3^{*, \#}$ \\
\hline Ionized Ca $(\mathrm{mmol} / \mathrm{L})$ & $1.19 \pm 0.095$ & $1.22 \pm 0.09$ & $1.17 \pm 0.11$ \\
\hline Phosphorus (mg/dl) & $2.1 \pm 0.65$ & $2.11 \pm 0.60$ & $2.09 \pm 0.65$ \\
\hline BMD (L1-L4) (g/cm²) & $1.13 \pm 0.11$ & $1.01 \pm 0.09$ & $0.78 \pm 0.07^{*, \#}$ \\
\hline BMD femoral neck $\left(\mathrm{g} / \mathrm{cm}^{2}\right)$ & $0.89 \pm 0.08$ & $0.84 \pm 0.09$ & $0.68 \pm 0.08^{*, \#}$ \\
\hline
\end{tabular}

Data presented as Mean $\pm \mathrm{SEM}^{*}$ Significantly different from group I by ANOVA P $<0.05^{\#}$ Significantly different from group II by ANOVA P $<0.05$.

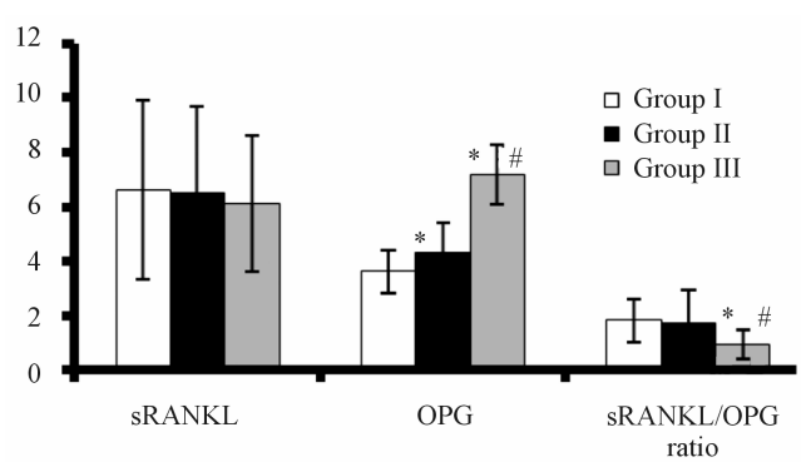

Figure 1. The levels of sRANKL and OPG (pg/ml) and SRANKL/OPG ratio in the control subjects and diabetic postmenopausal women with and without osteoporosis. Data presented as Mean \pm SEM, "Significantly different from group I by ANOVA, "Significantly different from group II by ANOVA $P<0.05$.

about $7 \%$ compared to control group. The ratio of sRANKL/OPG among diabetic osteoporotic postmenopausal women showed significant decrease by about $50 \%$, while no significant change was observed in group II compared to control group (Figure 1).

The result showed that, the diabetic postmenopausal women with osteoporosis has a significantly decreased IGF-1 levels by about $61 \%$, while diabetic women without osteoporosis has a significant decrease by about $30 \%$ compared to control values (Figure 2). Group III who suffer from osteoporosis showed a significant decrease in osteocalcin level by about $27 \%$ and those who are not osteoporotic show no significant change compare to control group (Figure 2).

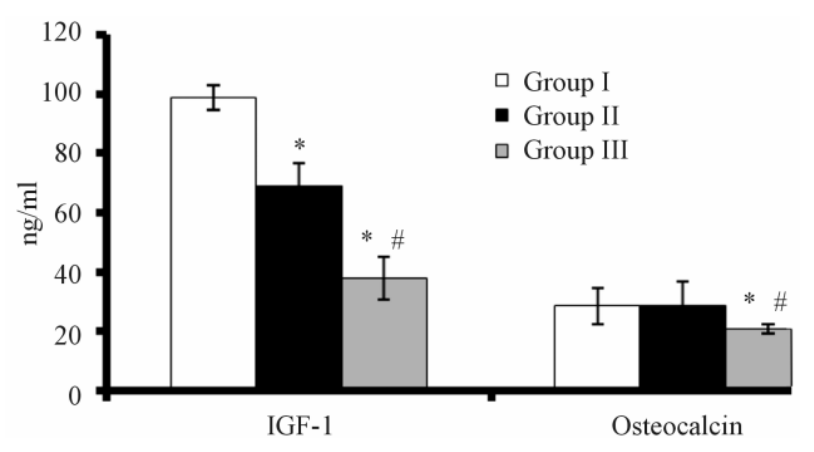

Figure 2. The levels of IGF-1 and osteocalcin in the control subjects and diabetic postmenopausal women with and without osteoporosis. Data presented as Mean \pm SEM. "Significantly different from group I by ANOVA, "Significantly different from group II by ANOVA $P<0.05$.

The correlation studies indicated that in group III the level of IGF-1 is significantly negatively correlated with OPG ( $r=-0.887, p<0.0001$, Figure 3), and fasting glucose $(r=-0.584, p=0.001$, Figure 4) and positively correlated with sRANKL $(r=0.893, p<0.001$, Figure 5), sRANKL/OPG ratio $(\mathrm{r}=0.859, \mathrm{p}<0.001$, Figure 6), lumber BMD ( $\mathrm{r}=0.828, \mathrm{p}=0.0001$, Figure 7) and femoral BMD ( $r=0.0807, p=0.0001$, Figure 8). Also, in the same group the level of osteocalcin show a significant positive correlation with OPG $(r=0.524, p=0.003$, Figure 9) and negative correlation with sRANKL $(\mathrm{r}=$ $-0.452, \mathrm{p}=0.012$, Figure 10).

\section{Discussion}

Postmenopause is a strong risk factor for the develop- 


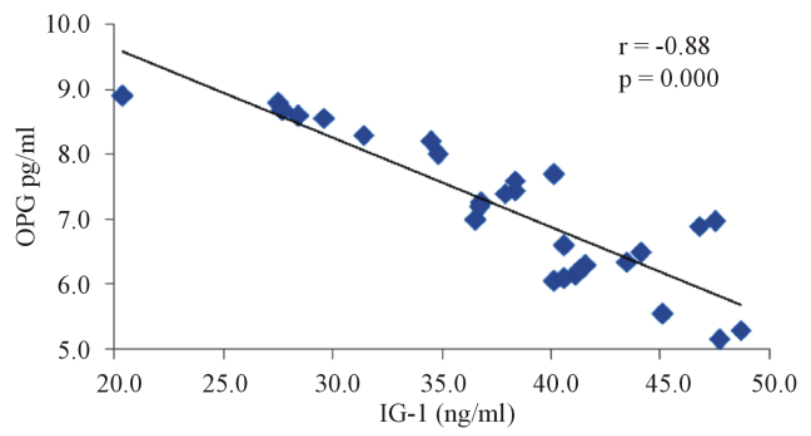

Figure 3. The correlation between serum IGF-1 and OPG in diabetic osteoporotic postmenopausal women.

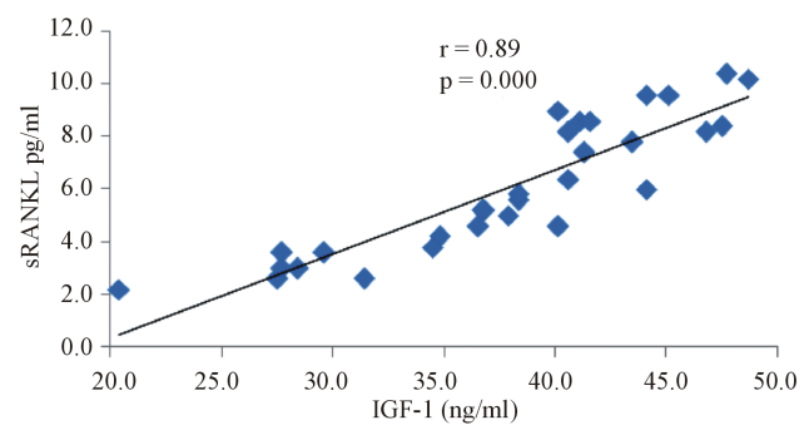

Figure 4. The correlation between serum IGF-1 and FBG in diabetic osteoporotic postmenopausal women.

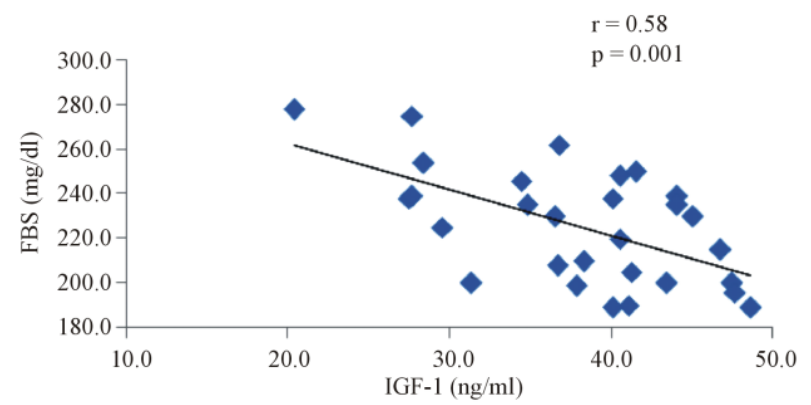

Figure 5. The correlation between serum IGF-1 and sRAN$\mathrm{KL}$ in diabetic osteoporotic postmenopausal women.

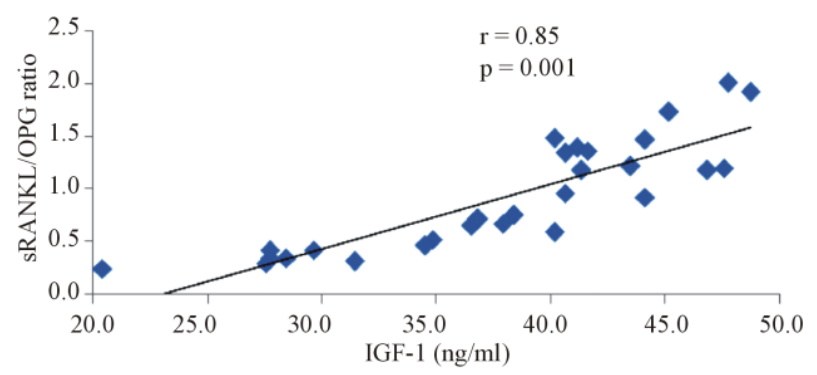

Figure 6. The correlation between serum IGF-1 and sRANKL/OPG in diabetic osteoporotic postmenopausal women.

ment of osteoporosis [17]. The osteoporosis in post- menopausal diabetic women is complex process and a matter of debates, so this study was conducted to eva-

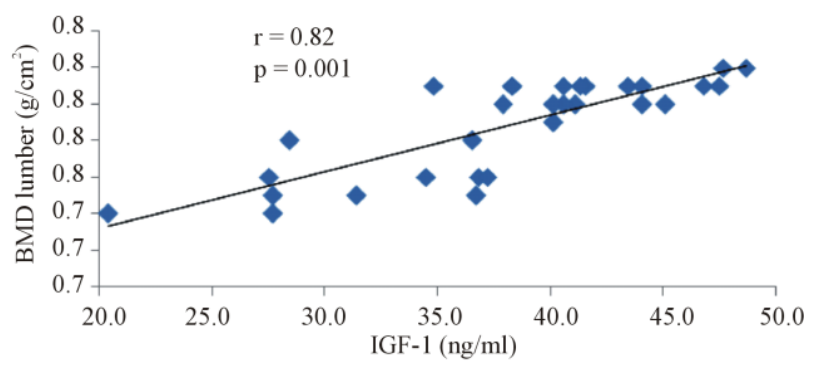

Figure 7. The correlation between serum IGF-1 levels and lumber BMD in diabetic osteoporotic postmenopausal women.

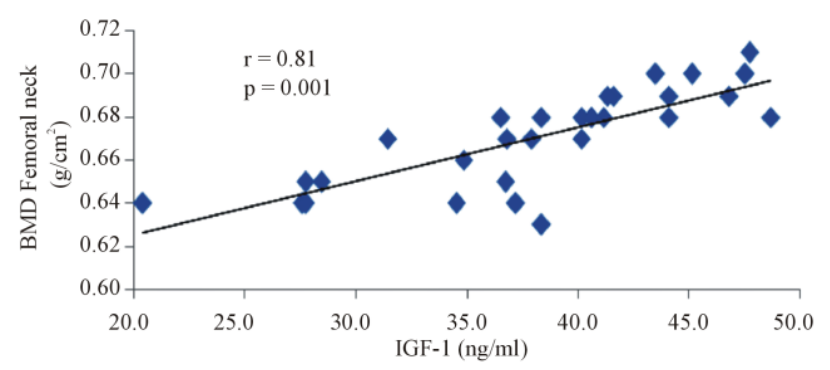

Figure 8. The correlation between serum IGF-1 and femoral neck BMD in diabetic osteoporotic postmenopausal women.

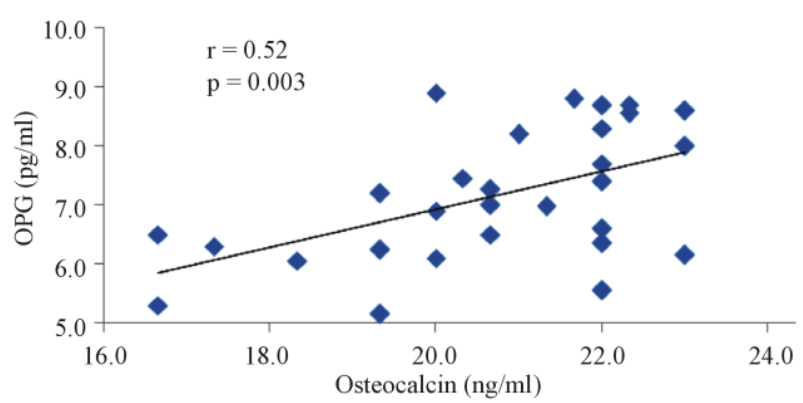

Figure 9. The correlation between serum OPG levels and osteocalcin in diabetic osteoporotic postmenopausal women.

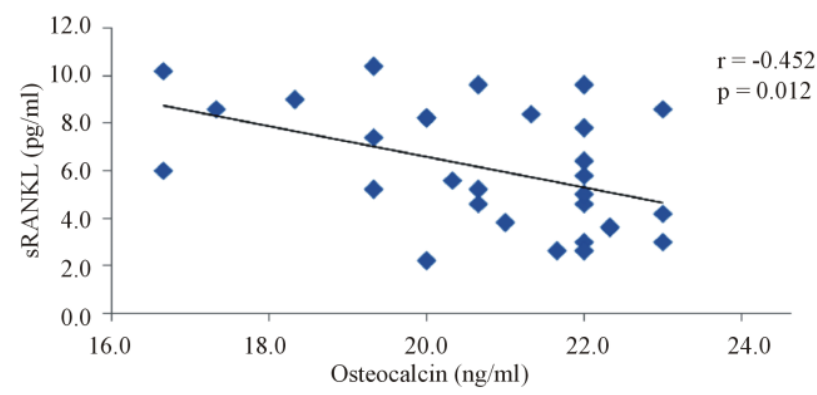

Figure 10. The correlation between serum sRANKL levels and osteocalcin in diabetic osteoporotic postmenopausal women.

luate osteoporosis in postmenopausal women with Type $2 \mathrm{DM}$ and to explore IGF-1and osteocalcin as a markers for assessing bone remodeling in those subjects. 
Our results indicated that IGF-1 is decreased by about quarter in postmenopausal diabetic women without osteoporosis, while those diabetic women with osteoporosis show about $60 \%$ decrease in IGF-1 compared to control subjects. Also, IGF-1 is significantly negatively correlated with fasting blood glucose level in postmenopausal women with osteoporosis. In line with our results, Zhao et al. 2008 [18] and other studies demonstrated that serum IGF-1 levels were significantly and negatively associated with age and its level is lower in postmenopausal women than in premenopausal women. Low levels of IGF-1 may increase the risk of fractures as indicated that low level of IGF-1 predicted osteoporotic fractures independently of the BMD [19,20]. It was also found that IGF-1 level decreased after menopause or with estrogen deficiency $[21,22]$. All age-related decrease of IGF-1 may correlate with age-related bone loss or osteoporosis.

The role of IGF-1 as an important regulator of bone formation is well established, however, its effects on bone resorption is limited and conflicting [23,24]. Also, its impact on the balance of the two peptides produced by osteoblasts, OPG and RANKL, is under investigation. Thus we aimed from this study to analyze the relationship of serum concentration of IGF-1 with OPG, RANKL and sRANKL/OPG ratio. The result indicated that while serum OPG levels are elevated in diabetic women especially those with osteoporosis the levels of sRANKL tend to decrease insignificantly in the same group. The sRANKL/OPG ratio which represents sRANKL bioactivity index show a significant decrease in diabetic postmenopausal women, the decrease is more prominent in those women with osteoporosis.

In line with our result many studies reported an increased level of OPG in postmenopausal osteoporosis [25-27]. Another studies proved that OPG is increased in diabetic women than non-diabetic subjects and its level is correlated with fructosamine concentration [28]. Elevated levels of plasma OPG were reported in newly diagnosed Type 2 diabetic patients and these levels were associated with endothelium-dependent arterial dilation [29]. Another recent population based study found significantly higher levels of serum OPG in postmenopausal Type 2 women than healthy postmenopausal women [30].

The study of Abrahamsen et al. 2005, indicate a decreased SRANKL and increased OPG levels and decreased sRANKL/OPG ratio with menopause [25]. This suggests that change in the circulating amounts of sRANKL with menopause and age may constitute a compensatory mechanism in response to increased bone resorption. The results of increasing levels of OPG and decreasing sRANKL/OPG ratio in osteoporotic women appear to be conflict, as OPG is a decoy receptor for RANKL so it should considered as a protective mecha- nism so how we can explain these results.

From the available literatures and our study we can explain these results through different approaches. First: many years ago, the experimental data and clinical observations about OPG-sRANKL system appears however conflicting. OPG-deficient mice develop sever osteoporosis with multiple fractures and calcification of the aorta and renal arteries [31]. On the contrary in patients with osteoporosis, hypertension and cardiovascular diseases the serum levels of OPG are higher [32-34]. These finding suggest that elevated OPG may represent an insufficient compensatory self-defensive mechanism to prevent bone resorption and vascular damage. Second: The serum level of OPG does not necessarily reflect the actual concentration in the bone microenvironment because tissues other than bone (e.g. lung, kidney, endothelial cells and arterial smooth muscle cells) produce OPG [35]. Third: The available ELISA assay used for the measurement of serum OPG detect both monomeric and dimeric OPG as well as OPG bound to circulating sRANKL. It's known that the OPG binding to sRANK reduce its clearance and so results in increased serum levels of OPG [28]. Fourth: sRANKL is membrane anchored molecule which can be cleaved from the cell surface as soluble sRANKL [36]. The RANKL test kits available now detect SRANKL in biological fluid such as serum. Whether the amount and activities of SRANKL are related to their membrane bound form is still uncertain.

Due to those conflict about the level of OPG bone turnover marker in osteoporotic diabetic patients makes them misleading and confusing. So other markers should be explored, in our study we explore the association of these parameter with IGF-1 (as new marker). The correlation studies indicted a strong significant negative correlation between serum levels of IGF-1 and OPG in postmenopausal diabetic women with osteoporosis. With osteoporosis, while its level in the same group shows a strong positive correlation with BMD at lumber and femoral neck, sRANKL and sRANKL/OPG ratio.

In line with our results the in vitro study of Rubin et al. 2002 indicated that IGF-1 increased sRANKL and decreased OPG expression in mouse stromal cells [37]. Furthermore, they found that IGF-1 treatment to postmenopausal women decreased OPG expression. Also in accordance with the obtained data Zhao et al. 2008 showed that serum IGF-1 is negatively correlated with serum OPG and positively with sRANKL/OPG ratio and sRANKL in postmenopausal women [18]. When they divided the postmenopausal women into normal, osteopenic and osteoporotic groups, it was found that serum IGF-1 levels in osteoporotic women were more than those in normal women but no significant difference was found in OPG and RANKL among the groups. These studies with our study confirmed that the IGF-1 level 
may act as an indicator of bone remodeling in post- menopausal women with Type 2 diabetes.

\section{Conclusions}

The present study indicated that serum osteocalcin level showed no significant change in postmenopausal diabetic women without osteoporosis, while those women with osteoporosis showed a significant decline compared to healthily control non-diabetic postmenopausal women (Figure 2). In accordance with these results, Im et al. 2008 demonstrated that the serum level of osteocalcin was lower and found to be an independent risk factor associated with glucose and glycated hemoglobin in postmenopausal women with Type 2 diabetes [38]. Also other studies indicated that, Type 1 and Type 2 diabetes were associated with lower levels of osteocalcin $[39,40]$. Also our correlation studies indicated that the serum level of Osteocalcin was negatively correlated with sRANKL and IGF-1, while positively correlated with OPG in postmenopausal diabetic women with osteoporosis.

From these results we can suggest that assessing the circulating levels of IGF-1 and osteocalcin may provide a very useful information for diagnosis or even prognosis of osteoporosis in Type 2 diabetic postmenopausal women because they greatly declines with osteoporosis in those patients.

\section{REFERENCES}

[1] U. Lerner, "Bone Remodeling in Postmenopausal Osteoporosis," Journal of Dental Research, Vol. 85, No. 7, 2006, pp. 584-595. doi:10.1177/154405910608500703

[2] P. Downey and M. Siegel, "Bone Biology and the Clinical Implications for Osteoporosis," Physical Therapy, Vol. 86 , No. 1, 2006, pp. 77-91. doi:10.1016/j.coph.2005.06.005

[3] P. Kostenuik, "Osteoprotegerin and RANKL Regulate Bone Resorption, Density, Geometery and Strength," Current Opinion in Pharmacology, Vol. 5, No. 6, 2005, pp. 618-625.

[4] B. Boyce and L. Xing, "Biology of RANK, RANKL, and Osteoprotegerin," Arthritis Research \& Therapy, Vol. 9, Suppl. 1, 2007, p. S1. doi:10.1186/ar2165

[5] E. Canalis, "Insulin-Like Growth Factors and Osteoporosis," Bone, Vol. 21, No. 3, 1997, pp. 215-216. doi:10.1016/S8756-3282(97)00150-6

[6] M. Zhang, S. Xuan, M. Bouxsein, et al., "OsteoblastSpecific Knockout of the Insulin-Like Growth Factor (IGF) Receptor Gene Reveals an Essential Role of IGF Signaling in Bone Matrix Mineralization," The Journal of Biological Chemistry, Vol. 277, No. 46, 2002, pp. 4400544012. doi:10.1074/jbc.M208265200

[7] T. Dowd, J. Rosen and C. Gundberg, "The Three-Dimensional Structure of Bovine Calcium Ion-Bound Os- teocalcin Using 1H NMR Spectroscopy," Biochemistry, Vol. 42, No. 25, 2003, pp. 7769-7779.

doi: $10.1021 / \mathrm{bi034470s}$

[8] N. Lee, H. Sowa, E. Hinoi, et al., "Endocrine Regulation of Energy Metabolism by the Skeleton," Cell, Vol. 130, No. 3, 2007, pp. 456-469. doi:10.1016/j.cell.2007.05.047

[9] K. Wongdee and N. Charoenphandhu, "Osteoporosis in Diabetes Mellitus: Possible Cellular and Molecular Mechanisms," World Journal of Diabetes, Vol. 2, No. 3, 2011, pp. 41-48. doi:10.4239/wjd.v2.i3.41

[10] T. Yamaguchi, "Bone Fragility in Type 2 Diabetes Mellitus," World Journal of Orthopaedics, Vol. 1, No. 1, 2010, pp. 3-9. doi:10.5312/wjo.v1.i1.3

[11] M. Petit, M. Paudel, B. Taylor, et al., "Bone Mass and Strength in Older Men with Type 2 Diabetes: The Osteoporotic Fractures in Men Study," Journal of Bone and Mineral Research, Vol. 25, No. 2, 2010, pp. 285-291. doi:10.1359/jbmr.090725

[12] T. Yamaguchi, I. Kanazawa, M. Yamamoto, et al., "Associations between Components of the Metabolic Syndrome versus Bone Mineral Density and Vertebral Fractures in Patients with Type 2 Diabetes," Bone, Vol. 45, No. 2, 2009, pp. 174-179. doi:10.1016/j.bone.2009.05.003

[13] S. Yaturu, S. Humphrey, C. Landry and S. Jain, "Decreased Bone Mineral Density in Men with Metabolic Syndrome Alone and with Type 2 Diabetes," Medical Science Monitor, Vol. 15, No. 1, 2009, pp. 5-9.

[14] P. Trinder, "Determination of Glucose in Blood Using Glucose Oxidase with an Alternative Oxygen Acceptor," Annals of Clinical Biochemistry, Vol. 6, No. 2, 1969, pp. 24-27.

[15] E. Matteucci, V. Cinapri, L. Rossi, et al., "Glycated Hemoglobin Measurement: Intermethod Comparison," Diabetes, Nutrition \& Metabolism, Vol. 14, No. 4, 2001, pp. 217-219.

[16] S. Laxmayya, P. Sandhya and M. Umesh, "Serum Calcium Measurement: Total versus Free (Ionized) Calcium" Indian Journal of Clinical Biochemistry, Vol. 20, No. 2, 2005, pp. 158-161. doi:10.1007/BF02867418

[17] P. Schnatz, K. Marakovits and D. O’Sullivan, “Assessment of Postmenopausal Women and Significant Risk Factors for Osteoporosis," Obstetrical \& Gynecological Survey, Vol. 65, No. 9, 2010, pp. 591-596. doi:10.1097/OGX.0b013e3181fc6d30

[18] H. Zhao, J. Liu, G. Ning, et al., "Relationships between Insulin-Like Growth Factor-I (IGF-I) and OPG, RANKL, Bone Mineral Density in Healthy Chinese Women," Osteoporosis International, Vol. 19, No. 2, 2008, pp. 221226. doi:10.1007/s00198-007-0440-y

[19] P. Garnero, E. Sornay-Rendu and P. Delmas, "Low Serum IGF-1 and Occurrence of Osteoporotic Fractures in Postmenopausal Women," Lancet, Vol. 355, No. 9207, 2000, pp. 898-899. doi:10.1016/S0140-6736(99)05463-X

[20] I. Kanazawa, T. Yamaguchi, M. Yamamoto, et al., "Serum Insulin-Like Growth Factor-I level is Associated with the Presence of Vertebral Fractures in Postmenopausal Women with Type 2 Diabetes Mellitus," Osteo- 
porosis International, Vol. 18, No. 12, 2007, pp. 16751681. doi:10.1007/s00198-007-0430-0

[21] C. Posaci, S. Altunyurt, H. Islekel and A. Onvural, "Effects of HRT on Serum Levels of IGF-I in Postmenopausal Women," Maturitas, Vol. 40, No. 1, 2001, pp. 6974. doi:10.1016/S0378-5122(01)00230-4

[22] E. Poehlman, M. Toth, P. Ades, C. Rosen, "Menopause Associated Changes in Plasma Lipids, Insulin-Like Growth Factor-I, and Blood Pressure: A Longitudinal Study," European Journal of Clinical Investigation, Vol. 27, No. 4, 1997, pp. 322-326. doi:10.1046/j.1365-2362.1997.1160662.x

[23] S. Yakar, C. J. Rosen, "From Mouse to Man: Redefining the Role of Insulin-Like Growth Factor-I in the Acquisition of Bone Mass," Experimental Biology and Medicine (Maywood), Vol. 228, No. 3, 2003, pp. 245-252.

[24] T. Ueland, "GH/IGF-I and Bone Resorption in Vivo and in Vitro," European Journal of Endocrinology, Vol. 152, No. 3, 2005, pp. 327-332. doi:10.1530/eje.1.01874

[25] B. Abrahamsen, J. Hjelmborg, P. Kostenuik, et al., "Circulating Amounts of Osteoprotegerin and RANKL: Genetic Influence and Relationship with BMD Assessed in Female Twins" Bone, Vol. 36, No. 4, 2005, pp. 727-735. doi:10.1016/j.bone.2004.12.015

[26] S. Trofimov, I. Pantsulaia, E. Kobyliansky and G. Livshits, "Circulating Levels of Receptor Activator of $\mathrm{Nu}-$ clear Factor $\kappa \mathrm{B}$ Ligand/Osteoprotegerin/Macrophage-Colony Stimulating Factor in a Presumably Healthy Human Population," European Journal of Endocrinology, Vol. 150, No. 3, 2004, pp. 305-311. doi:10.1530/eje.0.1500305

[27] D. Vega, N. Maalouf and K. Sakhaee, "CLINICAL Review \#: the Role of Receptor Activator of Nuclear FactorKappaB (RANK)/RANK Ligand/Osteoprotegerin: Clinical Implications," The Journal of Clinical Endocrinology \& Metabolism, Vol. 92, No. 12, 2007, pp. 4514-4521. doi:10.1210/jc.2007-0646

[28] W. Browner, L. Lui and S. Cummings, "Association of Serum Osteoprotegerin Levels with Diabetes, Strike, Bone Density, Fractures and Mortality in Elderly Women," The Journal of Clinical Endocrinology \& Metabolism, Vol. 86, No. 2, 2001, pp. 631-637. doi:10.1210/jc.86.2.631

[29] G. D. Xiang, L. Xu, L. S. Zhao, et al., "The Relationship between Plasma Osteoprotegerin and Endothelium-Dependent Arterial Dilation in Type 2 Diabetes," Diabetes, Vol. 55, No. 7, 2006, pp. 2126-2131. doi: $10.2337 / \mathrm{db} 06-0231$

[30] I. Nabipour, M. Kalantarhormozi, B. Larijani, et al., "Osteoprotegerin in Relation to Type 2 Diabetes Mellitus and the Metabolic Syndrome in Postmenopausal Women," Metabolism, Vol. 59, No. 5, 2010, pp. 742-747. doi:10.1016/j.metabol.2009.09.019

[31] N. Bucay, I. Sarosi, C. Dunstan, et al., "OsteoprotegerinDeficient Mice Develop Early Onset Osteoporosis and
Arterial Calcification," Genes \& Development, Vol. 12, No. 9, 1998, pp. 1260-1268. doi:10.1101/gad.12.9.1260

[32] R. Mogelvang, S. Pedersen, A. Flyvbjerg, et al., "Comparison of Osteoprotegerin to Traditional Atherosclerotic Risk Factors and High-Sensitivity C-Reactive Protein for Diagnosis of Atherosclerosis," American Journal of Cardiology, Vol. 109, No. 4, 2012, pp. 515-520. doi:10.1016/j.amjcard.2011.09.043

[33] B. Guldiken, S. Guldiken, B. Turgut, et al., "Serum Osteoprotegerin Levels in Patients with Acute Atherothrombotic Stroke and Lacunar Infarct," Thrombosis Research, Vol. 120, No. 4, 2007, pp. 511-516. doi:10.1016/i.thromres.2006.12.004

[34] S. Ziegler, S. Kudlacek, A. Luger and E. Minar, "Osteoprotegerin Plasma Concentration Correlates with Severity of Peripheral Artery Disease," Arteriosclerosis, Vol. 182, No. 1, 2005, pp. 175-180. doi:10.1016/j.atherosclerosis.2005.01.042

[35] P. Collin-Osdoby, L. Rothe, F. Anderson, et al., "Receptor Activator of NF- $\kappa \mathrm{B}$ and Osteoprotegerin Expression by Human Microvascular Endothelial Cells, Regulation by Inflammatory Cytokines, and Role in Human Osteoclastogenesis," The Journal of Biological Chemistry, Vol. 276, No. 23, 2001, pp. 20659-20672. doi:10.1074/jbc.M010153200

[36] L. Lum, B. Wong, R. Josien, J. Becherer, et al., "Evidence for a Role of a Tumor Necrosis Factor-Alpha (TNF-Alpha)-Converting Enzyme-Like Protease in Shedding of TRANCE, a TNF Family Member Involved in Osteoclastogenesis and Dentritic Cell Survival," The Journal of Biological Chemistry, Vol. 274, No. 19, 1999, pp. 13613-13618. doi:10.1074/jbc.274.19.13613

[37] J. Rubin, C. Ackert-Bicknell, L. Zhu, X. Fan, et al., "IGF-1 Regulates Osteoprotegerin (OPG) and Receptor Activator of Nuclear Factor $\kappa \mathrm{B}$ Ligand in Vitro and OPG in Vivo," The Journal of Clinical Endocrinology \& Metabolism, Vol. 87, No. 9, 2002, pp. 4273-4279. doi:10.1210/jc.2002-020656

[38] J. A. Im, B. P. Yu, J. Y. Jeon and S. H. Kim, "Relationship between Osteocalcin and Glucose Metabolism in Postmenopausal Women," Clinica Chimica Acta, Vol. 396, No. 1-2, 2008, pp. 66-69. doi:10.1016/j.cca.2008.07.001

[39] I. Kanazawa, T. Yamaguchi, Y. Tada, et al., "Serum Osteocalcin Level Is Positively Associated with Insulin Sensitivity and Secretion in Patients with Type 2 Diabetes," Bone, Vol. 48, No. 4, 2011, pp. 720-725. doi:10.1016/j.bone.2010.12.020

[40] L. McCabe, "Understanding the Pathology and Mechanisms of Type 1 Diabetic Bone Loss," Journal of Cellular Biochemistry, Vol. 102, No. 6, 2007, pp. 1343-1357. doi:10.1002/jcb. 21573 